18

\title{
Наноразмерные оксиды тантала, гафния и церия для монохроматических пучков фотонов и брахитерапии*
}

\author{
(C) В.Н. Морозов ${ }^{1,2}$, А.В. Белоусов ${ }^{1}$, Г.А. Крусанов ${ }^{3}$, М.А. Колыванова ${ }^{2}$, П.В. Кривошапкин ${ }^{4}$, \\ В.В. Виноградов ${ }^{4}$, А.П. Черняев ${ }^{1,3}$, А.А. Штиль ${ }^{4,5}$ \\ ${ }^{1}$ Московский государственный университет им. М.В. Ломоносова, фризический фракультет, \\ 119991 Москва, Россия \\ ${ }^{2}$ Государственный научный центр Российской Федерации - \\ Федеральный медицинский биофизический центр имени А.И. Бурназяна ФМБА России, \\ 123098 Москва, Россия \\ ${ }^{3}$ Научно-исследовательский институт ядерной физики им. Д.В. Скобельцина \\ Московского государственного университета им. М.В. Ломоносова, \\ 119991 Москва, Россия \\ ${ }^{4}$ Университет ИТМО, \\ 197101 Санкт-Петербург, Россия \\ ${ }^{5}$ Национальный медицинский исследовательский центр онкологии им. Н.Н. Блохина Минздрава России, \\ 115478 Москва, Россия \\ e-mail: morozov.v.n@mail.ru
}

Поступила в редакцию 15.02.2018 г.

Наночастицы элементов с высоким атомным номером способны увеличивать поглощенную дозу при их накоплении в опухолевых клетках. Количественной мерой эффекта радиосенсибилизации служит фактор увеличения дозы, отношение доз без наночастиц и в присутствии наночастиц. В настоящей работе были аналитически рассчитаны значения фактора увеличения дозы керамических наночастиц $\mathrm{Ta}_{2} \mathrm{O}_{5}, \mathrm{HfO}_{2}$ и $\mathrm{CeO}_{2}$ в комбинации с монохроматическим излучением рентгеновского диапазона энергий $(1-180 \mathrm{keV})$ и низкоэнергетичными источниками для брахитерапии ${ }^{103} \mathrm{Pd}$ (средняя энергия $\left.20.6 \mathrm{keV}\right),{ }^{125} \mathrm{I}(26.7 \mathrm{keV})$, ${ }^{131} \mathrm{Cs}(30.4 \mathrm{keV})$. Все наночастицы продемонстрировали высокие значения фактора увеличения дозы при концентрации $5 \mathrm{mg} / \mathrm{ml}$ как для монохроматического излучения, так и для брахитерапевтических источников. Максимальные значения 1.7 получены для наночастиц оксида тантала. В случае брахитерапевтических источников наибольшие значения фактора увеличения дозы от 1.48 до 1.67 были получены для наночастиц $\mathrm{Ta}_{2} \mathrm{O}_{5}$ и $\mathrm{HfO}_{2}$. Проведенные расчеты подтверждают перспективность использования керамических наночастиц в качестве модификаторов дозы для лучевой терапии.

DOI: $10.21883 /$ OS.2018.07.46274.48-18

\section{Введение}

Нанотехнологии имеют высокий потенциал использования в биомедицинских приложениях, в частности в лечении злокачественных опухолей. Уникальные свойства наноматериалов позволяют им находить применение в различных диагностических и терапевтических методах лечения онкозаболеваний [1]. В последнее время привлекает внимание возможность использования наночастиц для увеличения эффективности лучевого лечения злокачественных опухолей [2]. Наночастицы элементов с высокими атомными номерами $Z$, например висмута $\left(Z_{\mathrm{Bi}}=83\right)$, золота $\left(Z_{\mathrm{Au}}=79\right)$, серебра $\left(Z_{\mathrm{Ag}}=47\right)$ и пр., являются перспективными кандидатами для создания противоопухолевых радиосенсибилизаторов [3,4]. Кроме металлических наночастиц одним из многообещающих классов радиосенсибилизаторов являются керами-

* The 1st International School-conference for young researchers „Smart nanosystems for translation medicine“, November 28-29, 2017, St. Petersburg, Russia. ческие наночастицы, такие как оксиды тантала $\mathrm{Ta}_{2} \mathrm{O}_{5}$ $\left(Z_{\mathrm{Ta}}=73\right)[5,6]$, гафния $\mathrm{HfO}_{2}\left(Z_{\mathrm{Hf}}=72\right)$ [7] и церия $\mathrm{CeO}_{2}\left(Z_{\mathrm{Ce}}=58\right)[8]$.

Радиосенсибилизирующее действие наночастиц было исследовано с использованием рентгенотерапевтических аппаратов (до $300 \mathrm{keV}$ ), медицинских ускорителей электронов в режиме облучения фотонами (4-25 MeV), a также фотонных источников для брахитерапии [3]. Особенно интересными нанорадиосенсибилизаторы могут оказаться в таких перспективных направлениях лучевой терапии с монохроматическими или близкими к ним пучками фотонов, как Microbeam Radiation Therapy (MRT), Minibeam Radiation Therapy (MBRT) и Stereotactic Synchrotron Radiation Therapy (SSRT) [9].

B настоящей работе проведен аналитический расчет факторов усиления дозы наночастиц оксидов тантала, гафния и церия в комбинации с монохроматическим излучением с энергиями от 1 до $180 \mathrm{keV}$, а также с низкоэнергетичными фотонными источниками для брахитерапии ${ }^{103} \mathrm{Pd},{ }^{125} \mathrm{I}$ и ${ }^{131} \mathrm{Cs}$. 


\section{Материалы и методы}

Значения факторов усиления дозы (dose enhancement factor, DEF) для наночастиц оксидов тантала, гафния и церия $(5 \mathrm{mg} / \mathrm{ml})$ в зависимости от энергии первичных фотонов для случая монохроматического излучения, а также для трех низкоэнергетичных источников для брахитерапии были рассчитаны аналитически. Энергия первичных фотонов монохроматического излучения составляла $1-180 \mathrm{keV}$ с шагом $1 \mathrm{keV}$. Средние энергии источников для брахитерапии составили: ${ }^{103} \mathrm{Pd}-20.6 \mathrm{keV}$, ${ }^{125} \mathrm{I}-26.7 \mathrm{keV},{ }^{131} \mathrm{Cs}-30.4 \mathrm{keV}$ (линии с энергией менее $10 \mathrm{keV}$ не учитывались).

Фактор увеличения дозы DEF определяется как отношение поглощенной дозы в объеме интереса в присутствии наночастиц $D_{2}$ к поглощенной дозе в том же объеме при их отсутствии $D_{1}$ :

$$
\mathrm{DEF}=\frac{D_{2}}{D_{1}}
$$

Если известная поглощенная доза $D_{1}$ в некотором веществе „1“, то при выполнении условий электронного равновесия поглощенная доза $D_{2}$ в другом веществе „2“ в той же самой точке радиационного поля определяется выражением

$$
D_{2}=\frac{(\mu / \rho)_{2}}{(\mu / \rho)_{1}} D_{1}
$$

где $(\mu / \rho)_{i}-$ массовый коэффициент поглощения энергии фотонного излучения для $i$-го вещества. В случае монохроматического излучения и не очень больших размеров объема интереса условия электронного равновесия выполняются достаточно хорошо для того, чтобы ошибка определения поглощенной дозы не превышала $10 \%$. В случае полихроматических источников выражение (2) записывается в виде

$$
D_{2}=\frac{\sum_{i} E_{i} n_{i}\left(\frac{\mu\left(E_{i}\right)}{\rho}\right)_{2}}{\sum_{i} E_{i} n_{i}\left(\frac{\mu\left(E_{i}\right)}{\rho}\right)_{1}} D_{1}=\frac{\overline{\left(\frac{\mu}{\rho}\right)_{2}}}{\overline{\left(\frac{\mu}{\rho}\right)_{1}}} D_{1}=\overline{\left(\frac{\mu}{\rho}\right)_{1}^{2}} D_{1} .
$$

В (3) через $n_{i}$ обозначено количество фотонов с энергией $E_{i}$, испущенных в расчете на один распад радиоактивного ядра, а суммирование распространяется по всем энергиям фотонов, испускаемых при распаде (включая рентгеновское излучение после перестройки атомных оболочек). Окончательно получаем следующее выражение для фактора увеличения дозы фотонного излучения

$$
\mathrm{DEF}=\overline{\left(\frac{\mu}{\rho}\right)_{1}^{2}} .
$$

Значения массового коэффициента поглощения энергии фотонного излучения для различных химических элементов доступны на сайте базы данных NIST. Для сложного вещества, представляющего собой смесь различных химических элементов, массовый коэффициент поглощения может быть рассчитан как

$$
\frac{\mu_{e n}}{\rho}=\sum_{i} w_{i}\left(\frac{\mu_{e n}}{\rho}\right)_{i}
$$

где $\left(\frac{\mu_{e n}}{\rho}\right)_{i}-$ коэффициент передачи для $i$-го элемента в смеси, а $w_{i}$ - массовое содержание данного элемента в смеси.

\section{Результаты и обсуждение}

В клинической практике рентгеновское излучения с энергией до $300 \mathrm{keV}$ используются для лучевого лечения поверхностных опухолей, например рака кожи. В этой же области энергий излучения находятся низкоэнергетические источники для контактной лучевой терапии - брахитерапии. При взаимодействии электромагнитного излучения с веществом в данной области энергий доминирует фотоэлектрическое поглощение, сечение взаимодействия которого обладает сильной зависимостью от атомного номера $\left(\sigma \sim Z^{4-5}\right)$ вещества. Это приводит к различиям между массовыми коэффициентами ослабления энергии фотонного излучения (т.е. вероятности взаимодействия фотонов) исследуемых в настоящей работе наночастиц и мягких тканей (рис. 1). По этой причине именно в этой области энергий наночастицы элементов с высоким атомным номером продемонстрировали наибольшие значения увеличения дозы [10]. Перспективным методом лучевой терапии является использование монохроматических пучков рентгеновских фотонов, причем Stereotactic Synchrotron Radiation Therapy (SSRT) требует присутствия в опухоли контрастного агента [9], роль которого могут сыграть керамические наночастицы. Возможность использования

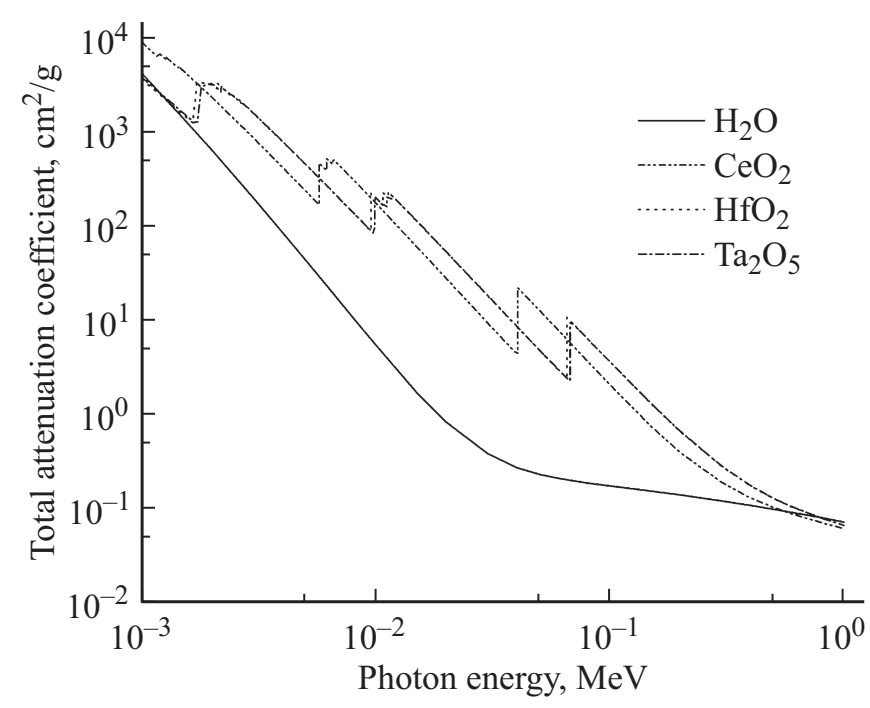

Рис. 1. Сравнение массовых коэффициентов ослабления для воды и оксидов тантала, гафния, церия. 


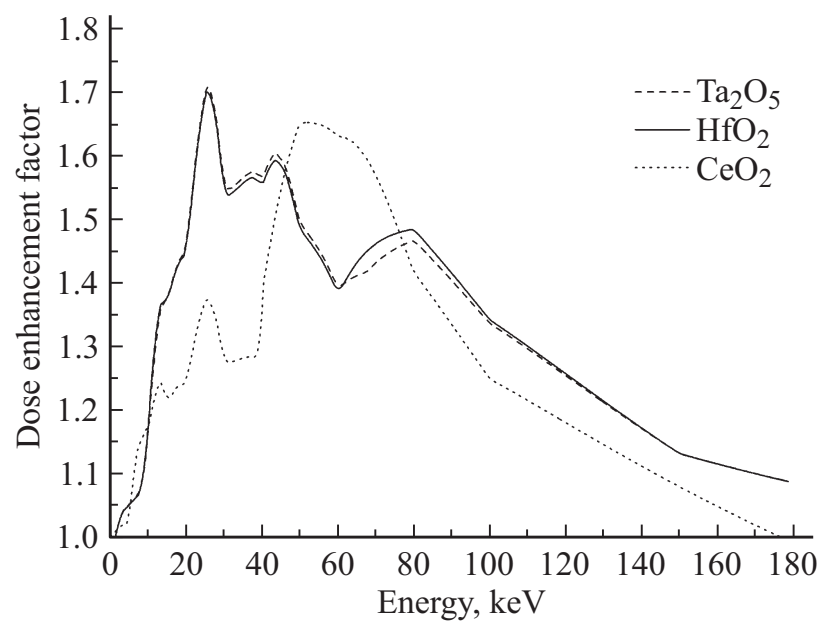

Рис. 2. Зависимость факторов усиления дозы (DEF) керамическими наночастицами от энергии монохроматического излучения.

наночастиц $\mathrm{Ta}_{2} \mathrm{O}_{5}$ в комбинации с монохроматическим излучением было исследовано методом Монте-Карло в работе [11].

Результаты аналитического расчета показывают, что использование керамических наночастиц в комбинации с монохроматическими пучками фотонов может значительно увеличить поглощенную дозу. На рис. 2 представлены зависимости фактора усиления дозы (DEF) от энергии первичных фотонов для каждого вида наночастиц $(5 \mathrm{mg} / \mathrm{ml})$. Максимальное значение DEF, равное $\sim 1.7$, было получено для наночастиц $\mathrm{Ta}_{2} \mathrm{O}_{5}$ при энергии фотонов $26 \mathrm{keV}$. Наночастицы оксидов тантала, гафния и церия показали усиление поглощенной дозы во всем диапазоне исследуемых энергий; однако их эффективность оказалась различной. Расчеты показывают, что предпочтительной стратегией для достижения максимального эффекта радиосенсибилизации было бы использование оптимальных наночастиц для каждой конкретной области монохроматических энергий излучения, а именно $1-9 \mathrm{keV}\left(\mathrm{CeO}_{2}\right), 10-24 \mathrm{keV}\left(\mathrm{HfO}_{2}\right)$, 25-45 keV $\left(\mathrm{Ta}_{2} \mathrm{O}_{5}\right), 46-75 \mathrm{keV} \quad\left(\mathrm{CeO}_{2}\right), \quad 76-150 \mathrm{keV}$ $\left(\mathrm{HfO}_{2}\right)$.

Полученные результаты в значительной степени согласуются с более ранними исследованиями, в которых с помощью метода Монте-Карло было установлено увеличение дозы монохроматического излучения в присутствии наночастиц оксидов гафния [7] и тантала [11].

Также установлено, что керамические наночастицы способны увеличивать дозу в комбинации с низкоэнергетичными источниками для брахитерапии. Значения факторов усиления дозы для трех различных источников для брахитерапии ${ }^{103} \mathrm{Pd},{ }^{125} \mathrm{I}$ и ${ }^{131} \mathrm{Cs}$ представлены в таблице. При концентрации $5 \mathrm{mg} / \mathrm{ml}$ наибольшее значение фактора усиления дозы достигается для наночастиц оксидов тантала и гафния. Различия между значениями DEF для $\mathrm{Ta}_{2} \mathrm{O}_{5}$ и $\mathrm{HfO}_{2}$ для всех трех источников по
Факторы усиления дозы (DEF) низкоэнергетичных фотонных источников для брахитерапии керамическими наночастицами при концентрации $5 \mathrm{mg} / \mathrm{ml}$

\begin{tabular}{l|c|c|c}
\hline Наночастицы & ${ }^{103} \mathrm{Pd}$ & ${ }^{125} \mathrm{I}$ & ${ }^{131} \mathrm{Cs}$ \\
\hline $\mathrm{Ta}_{2} \mathrm{O}_{5}$ & 1.48 & 1.67 & 1.55 \\
$\mathrm{HfO}_{2}$ & 1.48 & 1.66 & 1.54 \\
$\mathrm{CeO}_{2}$ & 1.26 & 1.35 & 1.28
\end{tabular}

сравнению с аналогичными значениями в случае оксида церия невелики, поэтому можно считать, что эффект наночастиц оксидов тантала и гафния одинаков. Значения DEF, которые показали керамические наночастицы, открывают широкие перспективы их использования в лучевой терапии, в том числе и при брахитерапевтических процедурах.

\section{Заключение}

В настоящей работе аналитически рассчитаны значения факторов увеличения дозы в присутствии керамических наночастиц $\mathrm{Ta}_{2} \mathrm{O}_{5}, \mathrm{HfO}_{2}$ и $\mathrm{CeO}_{2}$ в концентрации $5 \mathrm{mg} / \mathrm{ml}$ для случаев монохроматического фотонного излучения в диапазоне энергий от 1 до $180 \mathrm{keV}$, a также для трех низкоэнергетичных источников для брахитерапии ${ }^{103} \mathrm{Pd},{ }^{125} \mathrm{I},{ }^{131} \mathrm{Cs}$. Наибольшее значение $\mathrm{DEF} \sim 1.7$ было установлено для наночастиц $\mathrm{Ta}_{2} \mathrm{O}_{5}$ при энергии $26 \mathrm{keV}$. Наночастицы оксидов тантала и гафния показали наибольший эффект усиления дозы для всех трех исследованных низкоэнергетичных источников для брахитерапии от 1.48 до 1.67.

\section{Список литературы}

[1] Brigger I., Dubernet C., Couvreur P. // Adv. Drug. Deliv. Rev. 2002. V. 54. N 5. P. 631. doi 10.1016/j.addr.2012.09.006

[2] Hainfeld J.F., Slatkin D.N., Smilowitz H.M. // Phys. Med. Biol. 2004. V. 49. N 18. P. 309. doi 10.1088/0031-9155/49/18/N03

[3] Retif P., Pinel S., Toussaint M., Frochot C., Chouikrat R., Bastogne T., Barberi-Heyob M. // Theranostics. 2015. V. 5. N 9. P. 1030. doi 10.7150/thno.11642

[4] Her S., Jaffray D.A., Allen C. // Adv. Drug. Deliv. Rev. 2017. V. 109. P. 84. doi 10.1016/j.addr.2015.12.012

[5] Brown R., Tehei M., Oktaria S., Briggs A., Stewart C., Konstantinov K., Rosenfeld A., Corde S., Lerch M. // Part. Part. Syst. Charact. 2014. V. 31. N 4. P. 500. doi 10.1002/ppsc.201300276

[6] Engels E., Lerch M., Tehei M., Konstantinov K., Guatelli S., Rosenfeld A., Corde S. // J. Phys. Conf. Ser. 2017. V. 777. N 1. doi 10.1088/1742-6596/777/1/012011

[7] Maggiorella L., Barouch G., Devaux C., Pottier A., Deutsch E., Bourhis J., Borghi E., Levy L. // Future Oncol. 2012. V. 8. N 9. P. 1167. doi 10.2217/fon. 12.96

[8] Chen F., Zhang X.H., Hu X.D., Zhang W., Lou Z.C., Xie L.H., Liu P.D., Zhang H.Q. // Int. J. Nanomedicine. 2015. V. 10. P. 4957. doi 10.2147/IJN.S82980 
[9] Bräuer-Krisch E., Adam J.F., Alagoz E., Bartzsch S., Crosbie J., DeWagter C., Dipuglia A., Donzelli M., Doran S., Fournier P., Kalef-Ezra J., Kock A., Lerch M., McErlean C., Oelfke U., Olko P., Petasecca M., Povoli M., Rosenfeld A., Siegbahn E.A., Sporea D., Stugu B. // Phys Med. 2015. V. 31. N 6. P. 568. doi 10.1016/j.ejmp.2015.04.016

[10] Rahman W.N., Bishara N., Ackerly T., He C.F., Jackson P., Wong C., Davidson R., Geso M. // Nanomedicine. 2009. V. 5. N 2. P. 136. doi $10.1016 /$ j.nano.2009.01.014

[11] Engels E., Corde S., McKinnon S., Incerti S., Konstantinov K., Rosenfeld A., Tehei M., Lerch M., Guatelli S. // Phys Med. 2016. V. 32 N 12. P. 1852.

doi 10.1016/j.ejmp.2016.10.024 